\title{
Design of Highly Isolated Compact Antenna Array for MIMO Applications
}

\author{
Tiancong Huang, Yantao Yu, and Lijun Yi \\ College of Communication Engineering, Chongqing University, Chongqing 400044, China \\ Correspondence should be addressed to Yantao Yu; yantaoyu@cqu.edu.cn
}

Received 4 June 2014; Revised 2 September 2014; Accepted 6 November 2014; Published 20 November 2014

Academic Editor: Dau-Chyrh Chang

Copyright (C) 2014 Tiancong Huang et al. This is an open access article distributed under the Creative Commons Attribution License, which permits unrestricted use, distribution, and reproduction in any medium, provided the original work is properly cited.

In order to achieve very high data rates in both the uplink and downlink channels, the multiple antenna systems are used within the mobile terminal as well as the base station of the future generation of mobile networks. When implemented in a size limited platform, the multiple antenna arrays suffer from strong mutual coupling between closely spaced array elements. In this paper, a rigorous procedure for the design of a 4-port compact planar antenna array with high port isolation is presented. The proposed design involves a decoupling network consisting of reactive elements, whose values can be obtained by the method of eigenmode analysis. Numerical results show the effectiveness of the proposed design approach in improving the port isolation of a compact four-element planar array.

\section{Introduction}

The multiple-input-multiple-output (MIMO) system has become a promising technique in existing and future generation of wireless communication systems. Exploiting multiple antennas at both the transmitting and receiving ends can linearly increase the channel capacity of the system without increasing its overall bandwidth and power [1]. Implementing multiple antennas at the fixed terminal is an easy work if space is not a primary concern [2]. However, the implementation of multiple antennas in a compact terminal involves challenging design tradeoffs [3]. This is due to the severe effects of mutual coupling among the closely spaced elements, which will cause significant system performance degradation [4]. For example, the high capacity offered by the MIMO system is reduced if the various signals at the receiver are correlated, where the correlation may arise from the mutual coupling between the transmitting and/or receiving antenna elements [5-7].

The mutual coupling problem in antenna arrays and MIMO systems has attracted various studies in recent years [8-10]. Antenna decoupling techniques can be used to facilitate a smaller antenna separation for a given set of performance requirements [11]. Signal processing techniques using different coupling matrices [12] may be applied to the received signal vectors from adaptive arrays to counter the effects of mutual coupling. The use of parasitic scatters is also reported to reduce mutual coupling of MIMO arrays [13, 14]. The defected ground structures can also be implemented to reduce the mutual coupling effect between elements [15], but these structures usually occupy large space. A neutralization line can be inserted between two antennas to cancel the mutual coupling by introducing some current and creating an additional electromagnetic field [16]. Another way to isolate highly coupled monopole antenna elements is based on the mode-decomposition method, in which a multiport network using $90^{\circ}$ or $180^{\circ}$ hybrid couplers is inserted between the antennas and their feeding ports [17]. It is also shown that the passive and lossless decoupling and matching networks can be used to transform the coupled antenna ports into independent and matched ports. The papers $[18,19]$ introduced the possibility of connecting a lossless network between the input ports and the antenna ports for isolation, but it works in the condition that the antenna mutual impedances should be purely reactive at the resonance frequency. The design of decoupling network for arrays with complex impedance matrices can be found in [20] for a two-element array. 


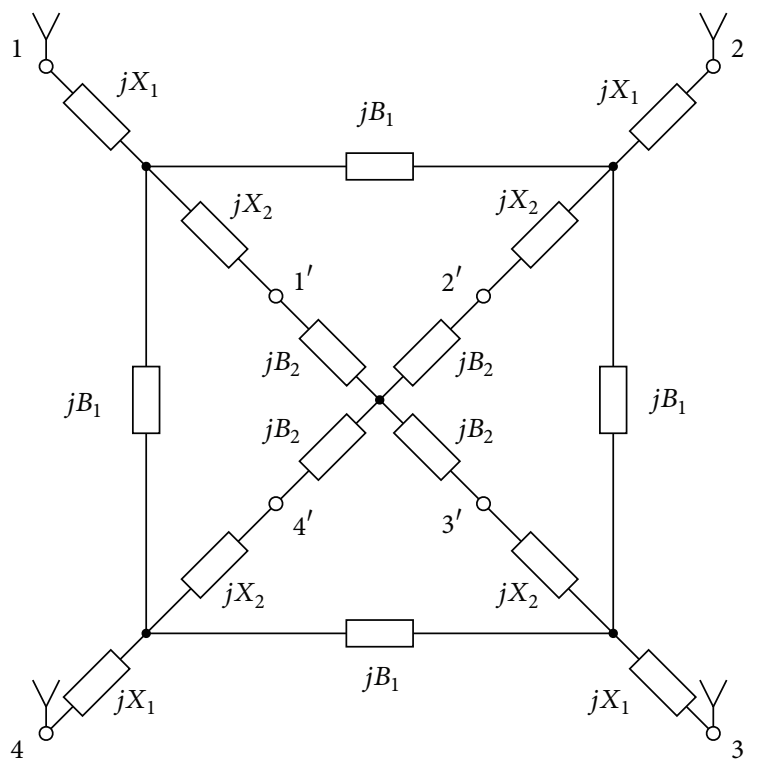

FIGURE 1: The decoupling network for a four-element antenna array.

In this paper, a rigorous design of decoupling network for a compact four-element symmetrical planar array is presented. The proposed decoupling network consists of serial- and parallel-connected reactive elements, the values of which can be calculated by solving a group of equations derived by using the eigenmode analysis method. The decoupled antenna ports can easily be matched using L-section matching circuit. Numerical results on a four-monopole array with an antenna spacing of 0.1 wavelength show that the compact array has highly isolated driving ports and it is suitable for MIMO applications.

\section{The Proposed Decoupling Network}

The proposed decoupling network for a four-element symmetric planar array is shown in Figure 1. It consists of two series sections with components $j X_{1}$ and $j X_{2}$ and two parallel sections with components $j B_{1}$ and $j B_{2}$. The ports 1 to 4 of the network are connected to the four ports of the array. The other four ports $1^{\prime}$ to $4^{\prime}$ are the driving ports of the decoupled antenna array.

The values of the components in the decoupling network can be calculated by using the eigenmode analysis method [19]. The impedance matrix of the symmetrical array is

$$
\mathbf{Z}=\left[\begin{array}{llll}
Z_{11} & Z_{12} & Z_{13} & Z_{12} \\
Z_{12} & Z_{11} & Z_{12} & Z_{13} \\
Z_{13} & Z_{12} & Z_{11} & Z_{12} \\
Z_{12} & Z_{13} & Z_{12} & Z_{11}
\end{array}\right]
$$

The eigenvalues of the impedance matrix are given by $Z_{m a}=$ $r_{a}+j x_{a}=Z_{11}+2 Z_{12}+Z_{13}, Z_{m b}=r_{b}+j x_{b}=Z_{11}-$ $Z_{13}=Z_{m c}$, and $Z_{m d}=r_{d}+j x_{d}=Z_{11}-2 Z_{12}+Z_{13}$, while the corresponding orthogonal eigenvectors are $\mathbf{v}_{a}=$ $[1,1,1,1]^{T}, \mathbf{v}_{b}=[1,1,-1,-1]^{T}, \mathbf{v}_{c}=[1,-1,-1,1]^{T}$, and
$\mathbf{v}_{d}=[1,-1,1,-1]^{T}$. The equivalent circuits of the decoupling network for different eigenmodes are shown in Figure 2.

According to Figure 2, the modal admittances for the four modes as seen from the new driving ports $1^{\prime}$ to $4^{\prime}$ are obtained as

$$
\begin{gathered}
Y_{1}=\left(Z_{m a}+j X_{1}+j X_{2}\right)^{-1} \\
Y_{2}=Y_{3}=\left(\left(\left(Z_{m b}+j X_{1}\right)^{-1}+j 2 B_{1}\right)^{-1}+j X_{2}\right)^{-1}+j B_{2} \\
Y_{4}=\left(\left(\left(Z_{m d}+j X_{1}\right)^{-1}+j 4 B_{1}\right)^{-1}+j X_{2}\right)^{-1}+j B_{2} .
\end{gathered}
$$

It is noted that $Y_{2}=Y_{3}$ for modes $b$ and $c$. In the eigenmode analysis, the decoupling network decouples the ports of the array by matching all the modal admittances. The values of $X_{1}, B_{1}, X_{2}$, and $B_{2}$ can be obtained by solving the nonlinear equations $Y_{1}=Y_{2}=Y_{4}$.

Consider (3) and (4) first. It is obvious that $Y_{2}=Y_{4}$ results in

$$
\left(Z_{m b}+j X_{1}\right)^{-1}=\left(Z_{m d}+j X_{1}\right)^{-1}+j 2 B_{1}
$$

By evaluating the real and imaginary parts of (5), the values of $X_{1}$ and $B_{1}$ can be obtained as

$$
\begin{gathered}
X_{1}=\frac{\left(r_{d} x_{b}-r_{b} x_{d}\right) \pm \sqrt{r_{b} r_{d}} \sqrt{\left(r_{b}-r_{d}\right)^{2}+\left(x_{b}-x_{d}\right)^{2}}}{r_{b}-r_{d}}, \\
B_{1}=\frac{1}{2}\left(\frac{x_{d}+X_{1}}{r_{d}^{2}+\left(x_{d}+X_{1}\right)^{2}}-\frac{x_{b}+X_{1}}{r_{b}^{2}+\left(x_{b}+X_{1}\right)^{2}}\right) .
\end{gathered}
$$

With $X_{1}$ and $B_{1}$ having been calculated, let

$$
\begin{gathered}
Z_{m a}+j X_{1}=Z_{m a}^{\prime}=r_{a}^{\prime}+j x_{a}^{\prime}, \\
\left(\left(Z_{m b}+j X_{1}\right)^{-1}+j 2 B_{1}\right)^{-1}=Z_{m b}^{\prime}=r_{b}^{\prime}+j x_{b}^{\prime} ;
\end{gathered}
$$

then (2) and (3) can be rewritten as

$$
\begin{gathered}
Y_{1}=\left(Z_{m a}^{\prime}+j X_{2}\right)^{-1} \\
Y_{2}=\left(Z_{m b}^{\prime}+j X_{2}\right)^{-1}+j B_{2} .
\end{gathered}
$$

Similarly, set $Y_{1}=Y_{2}$ and evaluate the real and imaginary parts, respectively. The values of $X_{2}$ and $B_{2}$ can be calculated as

$$
\begin{gathered}
X_{2}=\frac{\left(r_{b}^{\prime} x_{a}^{\prime}-r_{a}^{\prime} x_{b}^{\prime}\right) \pm \sqrt{r_{a}^{\prime} r_{b}^{\prime}} \sqrt{\left(r_{a}^{\prime}-r_{b}^{\prime}\right)^{2}+\left(x_{a}^{\prime}-x_{b}^{\prime}\right)^{2}}}{r_{a}^{\prime}-r_{b}^{\prime}} \\
B_{2}=\frac{x_{b}^{\prime}+X_{2}}{r_{b}^{\prime 2}+\left(x_{b}^{\prime}+X_{2}\right)^{2}}-\frac{x_{a}^{\prime}+X_{2}}{r_{a}^{\prime 2}+\left(x_{a}^{\prime}+X_{2}\right)^{2}} .
\end{gathered}
$$

Then, the decoupled antenna array can easily be matched to the system impedance $Z_{0}$ using the L-section impedance matching network. 


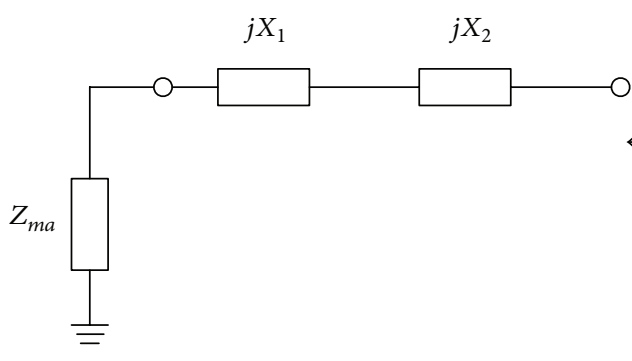

Mode $a$

(a)

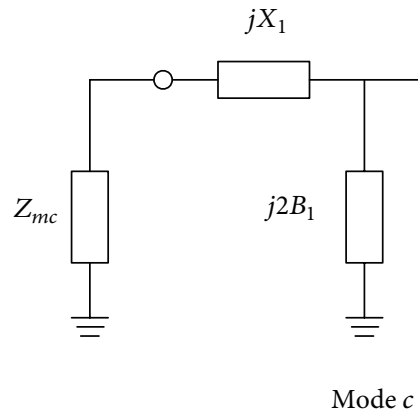

(c)

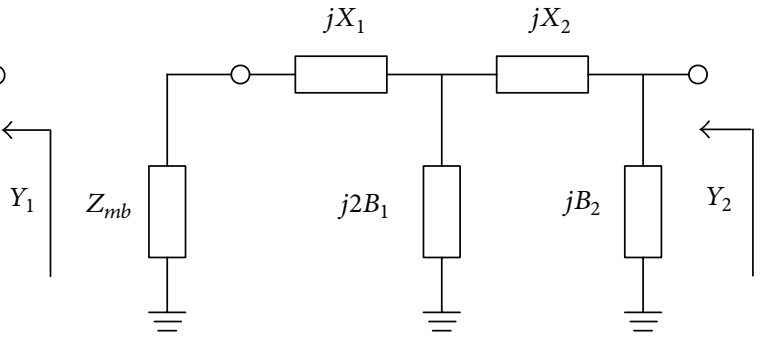

Mode $b$

(b)

FIgURE 2: The equivalent circuits for the different eigenmodes of a four-element array.

TABLE 1: Calculated components of the decoupling network.

\begin{tabular}{lcccc}
\hline Component & Solution 1 & Solution 2 & Solution 3 & Solution 4 \\
\hline$X_{1}$ & -25.506 & -25.506 & -19.365 & -19.365 \\
$B_{1}$ & -0.202 & -0.202 & 0.116 & 0.116 \\
$X_{2}$ & 3.601 & -8.755 & 13.921 & -5.498 \\
$B_{2}$ & 0.181 & -0.142 & 0.120 & -0.083 \\
\hline
\end{tabular}

\section{Example and Discussion}

As an illustration of the proposed decoupling theory, a compact four-monopole symmetrical planar array operating at $2.5 \mathrm{GHz}$ was designed. The monopole element has length of $28 \mathrm{~mm}$ and diameter of $1 \mathrm{~mm}$. The element separation is $12 \mathrm{~mm}$, which is about 0.1 wavelength at $2.5 \mathrm{GHz}$. The ground plane of the array is a circular metal disc with radius of $35 \mathrm{~mm}$. The EM simulation software Ansoft HFSS is used for simulation. The simulated scattering parameters of the monopole array are shown in Figure 3. It can be seen that the magnitude of the reflection coefficient $S_{11}$ of the array is about $-5 \mathrm{~dB}$ at $2.5 \mathrm{GHz}$ while the magnitudes of coupling coefficients $S_{12}$ and $S_{13}$ are about $-10 \mathrm{~dB}$ and $-13 \mathrm{~dB}$, respectively. Therefore, the ports of the compact antenna array are unmatched and highly coupled.

By exploiting the proposed decoupling procedure, the component values of the decoupling network are calculated and shown in Table 1. It is noted that there exist four groups of solutions. As an illustration, taking solution 1 and matching the decoupled ports by an L-section with parallel component $B_{3}=0.000205$ and serial component $X_{3}=46.515$, the

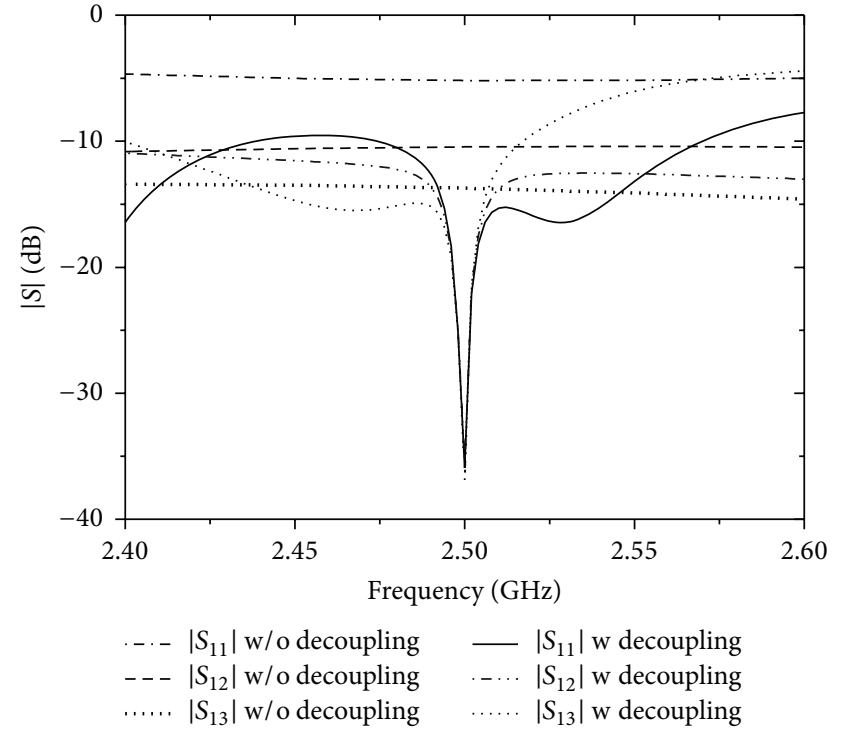

Figure 3: The $S$-parameters of the four-monopole array.

resulting scattering parameters of the decoupled and matched array are shown in Figure 3. It can be clearly seen that both the reflection coefficient and the coupling coefficient have been reduced to below $-30 \mathrm{~dB}$. The results clearly show the validity of the theory. Figure 4 depicts the simulated normalized azimuth radiation pattern of the decoupled array when port 1 is excited and the remaining ports are terminated with the matched loads. It is observed that the pattern is directional 


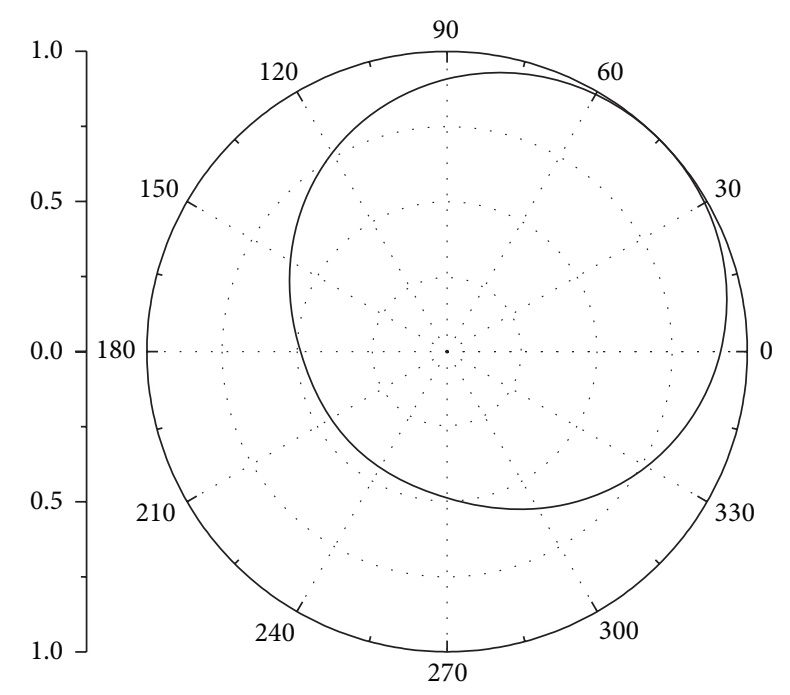

FIGURE 4: Normalized radiation pattern of the decoupled array with the excitation of port 1 .

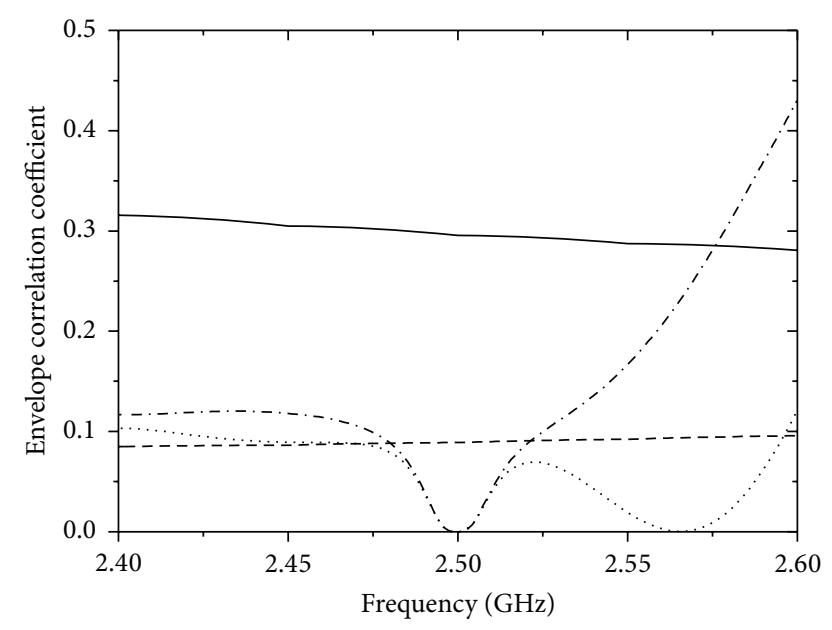

_ ECC b/t antenna elements 1 and $2 \mathrm{w} / \mathrm{o}$ decoupling

- - ECC b/t antenna elements 1 and $3 \mathrm{w} / \mathrm{o}$ decoupling

... - ECC b/t antenna elements 1 and $2 \mathrm{w}$ decoupling

...... ECC b/t antenna elements 1 and $3 \mathrm{w}$ decoupling

Figure 5: The envelope correlation coefficient of the array with and without the decoupling network.

and the array has strongest radiation at $\phi=45^{\circ}$. If port 2 or port 4 is excited, the radiation pattern will rotate about the $z$-axis by $\pm 90^{\circ}$, while, for the excitation of port 3 , a rotation of $180^{\circ}$ is needed.

As an important parameter for diversity performance, the envelope correlation coefficient (ECC) $\rho_{e}$ for the correlation between antenna elements $i$ and $j$ in an antenna array consisting of $N$ elements can be calculated using [21]

$$
\rho_{e}(i, j, N)=\frac{\left|\sum_{n=1}^{N} S_{i, n}^{*} S_{n, j}\right|^{2}}{\prod_{k=i, j}\left(1-\sum_{n=1}^{N} S_{k, n}^{*} S_{n, k}\right)} .
$$

Equation (10) follows the correlation equation of a twoantenna system in [22]. However, it is noted that the abovementioned correlation equations will fail in the extreme case of $S_{i j}=0$ and a solution to this problem has been provided in [23]. In this paper, $S_{i j} \neq 0$ and (10) can still provide a correlation analysis with reasonable accuracy. The calculated envelope correlations $\rho_{e}(1,2,4)$ and $\rho_{e}(1,3,4)$ of the fourmonopole array with and without the decoupling network are shown in Figure 5. It can be seen that the envelope correlations in the frequency band of interest have been reduced to much less than 0.1 , which means that the antenna array has good diversity gain and is suitable for MIMO systems.

\section{Conclusions}

The decoupling network consisting of simple reactive elements for a 4-element symmetrical planar array has been presented. The component values of the decoupling network can be calculated by using eigenmode analysis method. The simulated results show that high isolation between the driving ports of the compact array has been achieved and the compact design of the antenna system is suitable for applications in MIMO communications.

\section{Conflict of Interests}

The authors declare that there is no conflict of interests regarding the publication of this paper.

\section{Acknowledgments}

This work was supported by the National Natural Science Foundation of China (Grant 61101024), the Natural Science Foundation Project of CQ CSTC (Grant CSTC, 2011BB2070), and the Fundamental Research Funds for the Central Universities (Grants CDJZR12160013 and 106112014CDJZR165504).

\section{References}

[1] S. Zhang, B. K. Lau, Y. Tan, Z. Ying, and S. He, "Mutual coupling reduction of two PIFAs with a T-shape slot impedance transformer for MIMO mobile terminals," IEEE Transactions on Antennas and Propagation, vol. 60, no. 3, pp. 1521-1531, 2012.

[2] R. A. Bhatti, S. Yi, and S.-O. Park, "Compact antenna array with port decoupling for LTE-standardized mobile phones," IEEE Antennas and Wireless Propagation Letters, vol. 8, pp. 1430-1433, 2009.

[3] B. K. Lau, "Multiple antenna terminals," in MIMO: From Theory to Implementation, C. Oestges, A. Sibille, and A. Zanella, Eds., Academic Press, San Diego, Calif, USA, 2011.

[4] I. J. Gupta and A. A. Ksienski, "Effect of mutual coupling on the performance of adaptive arrays," IEEE Transactions on Antennas and Propagation, vol. 31, no. 5, pp. 785-791, 1983.

[5] R. Janaswamy, "Effect of element mutual coupling on the capacity of fixed length linear arrays," IEEE Antennas and Wireless Propagation Letters, vol. 1, pp. 157-160, 2002.

[6] P. N. Fletcher, M. Dean, and A. R. Nix, "Mutual coupling in multi-element array antennas and its influence on MIMO 
channel capacity," Electronics Letters, vol. 39, no. 4, pp. 342-344, 2003.

[7] M. J. Gans, "Channel capacity between antenna arrays-part I: sky noise dominates," IEEE Transactions on Communications, vol. 54, no. 9, pp. 1586-1592, 2006.

[8] J. W. Wallace and M. A. Jensen, "Mutual coupling in MIMO wireless systems: a rigorous network theory analysis," IEEE Transactions on Wireless Communications, vol. 3, no. 4, pp. 13171325, 2004.

[9] H. T. Hui, "A practical approach to compensate for the mutual coupling effect in an adaptive dipole array," IEEE Transactions on Antennas and Propagation, vol. 52, no. 5, pp. 1262-1269, 2004.

[10] M. L. Morris and M. A. Jensen, "Network model for MIMO systems with coupled antennas and noisy amplifiers," IEEE Transactions on Antennas and Propagation, vol. 53, no. 1, pp. 545-552, 2005.

[11] B. K. Lau and J. B. Andersen, "Simple and efficient decoupling of compact arrays with parasitic scatterers," IEEE Transactions on Antennas and Propagation, vol. 60, no. 2, pp. 464-472, 2012.

[12] H. Steyskal and J. S. Herd, "Mutual coupling compensation in small array antennas," IEEE Transactions on Antennas and Propagation, vol. 38, no. 12, pp. 1971-1975, 1990.

[13] Z. Li, Z. Du, M. Takahashi, K. Saito, and K. Ito, "Reducing mutual coupling of MIMO antennas with parasitic elements for mobile terminals," IEEE Transactions on Antennas and Propagation, vol. 60, no. 2, pp. 473-481, 2012.

[14] A. C. K. Mak, C. R. Rowell, and R. D. Murch, "Isolation enhancement between two closely packed antennas," IEEE Transactions on Antennas and Propagation, vol. 56, no. 11, pp. 3411-3419, 2008.

[15] Y. Jiang, Y. Yu, M. Yuan, and L. Wu, "A compact printed monopole array with defected ground structure to reduce the mutual coupling," Journal of Electromagnetic Waves and Applications, vol. 25, no. 14-15, pp. 1963-1974, 2011.

[16] C. Yang, J. Kim, H. Kim, J. Wee, B. Kim, and C. Jung, "Quadband antenna with high isolation MIMO and broadband SCS for broadcasting and telecommunication services," IEEE Antennas and Wireless Propagation Letters, vol. 9, pp. 584-587, 2010.

[17] T. I. Lee and Y. E. Wang, "Mode-based information channels in closely coupled dipole pairs," IEEE Transactions on Antennas and Propagation, vol. 56, no. 12, pp. 3804-3811, 2008.

[18] J. B. Andersen and H. H. Rasmussen, "Decoupling and descattering networks for antennas," IEEE Transactions on Antennas and Propagation, vol. 24, no. 6, pp. 841-846, 1976.

[19] H. J. Chaloupka, X. Wang, and J. C. Coetzee, "A superdirective 3-element array for adaptive beamforming," Microwave and Optical Technology Letters, vol. 36, no. 6, pp. 425-430, 2003.

[20] A. Diallo, C. Luxey, P. L. Thuc, R. Staraj, and G. Kossiavas, "Study and reduction of the mutual coupling between two mobile phone PIFAs operating in the DCS1800 and UMTS bands," IEEE Transactions on Antennas and Propagation, vol. 54, no. 11, pp. 3063-3074, 2006.

[21] J. Thaysen and K. B. Jakobsen, "Envelope correlation in (N, N) mimo antenna array from scattering parameters," Microwave and Optical Technology Letters, vol. 48, no. 5, pp. 832-834, 2006.

[22] S. Blanch, J. Romeu, and I. Corbella, "Exact representation of antenna system diversity performance from input parameter description," Electronics Letters, vol. 39, no. 9, pp. 705-707, 2003.

[23] X. Wang, H. D. Nguyen, and H. T. Hui, "Correlation coefficient expression by S-parameters for two omni-directional MIMO antennas," in Proceedings of the IEEE International Symposium on Antennas and Propagation and USNC/URSI National Radio Science Meeting (APSURSI '11), pp. 301-304, Spokane, Wash, USA, July 2011. 

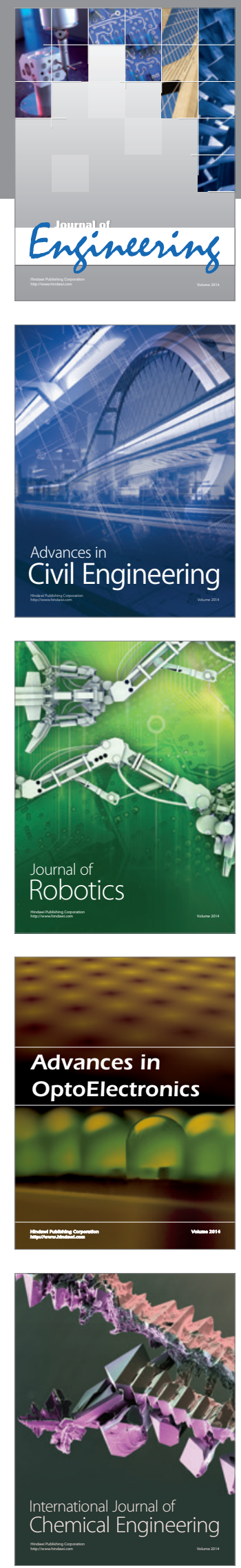

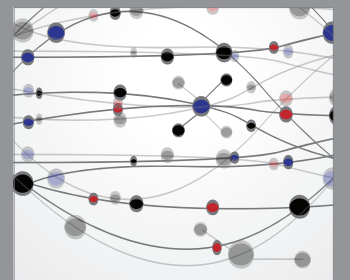

The Scientific World Journal
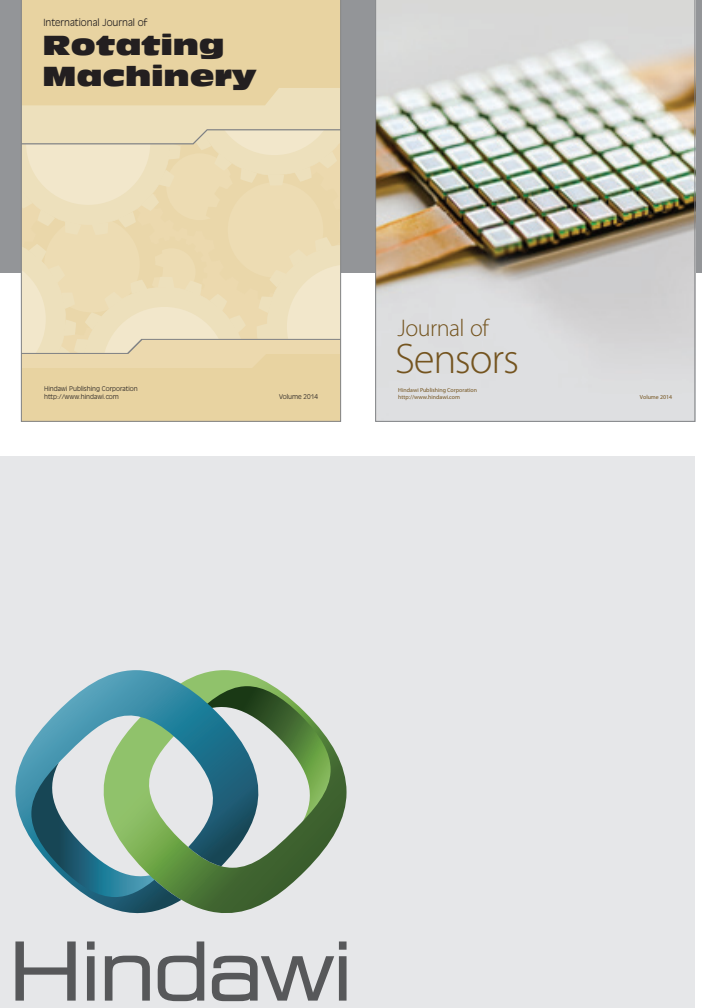

Submit your manuscripts at http://www.hindawi.com
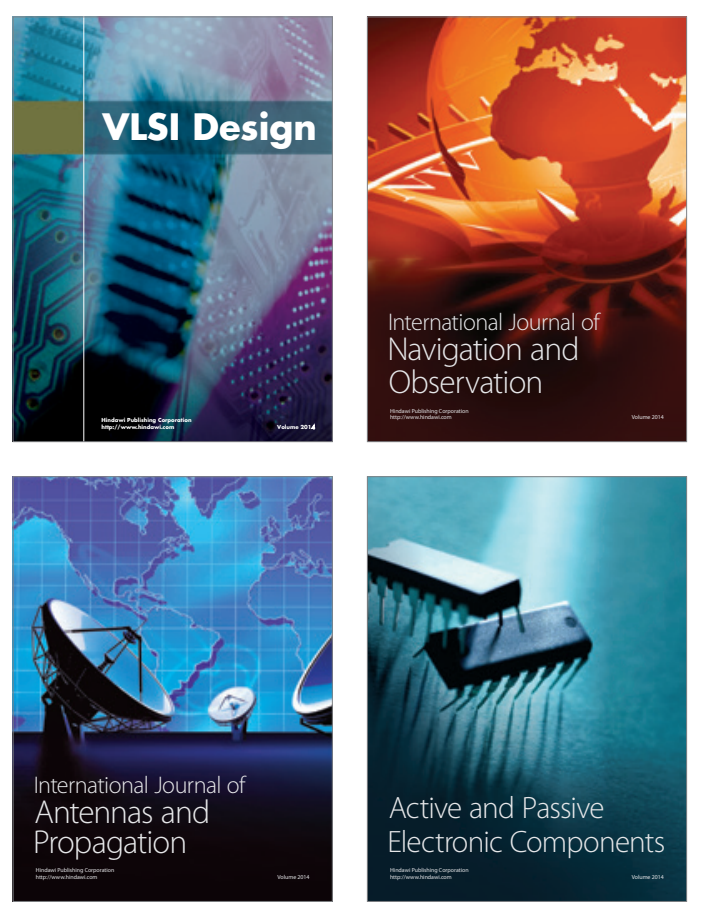
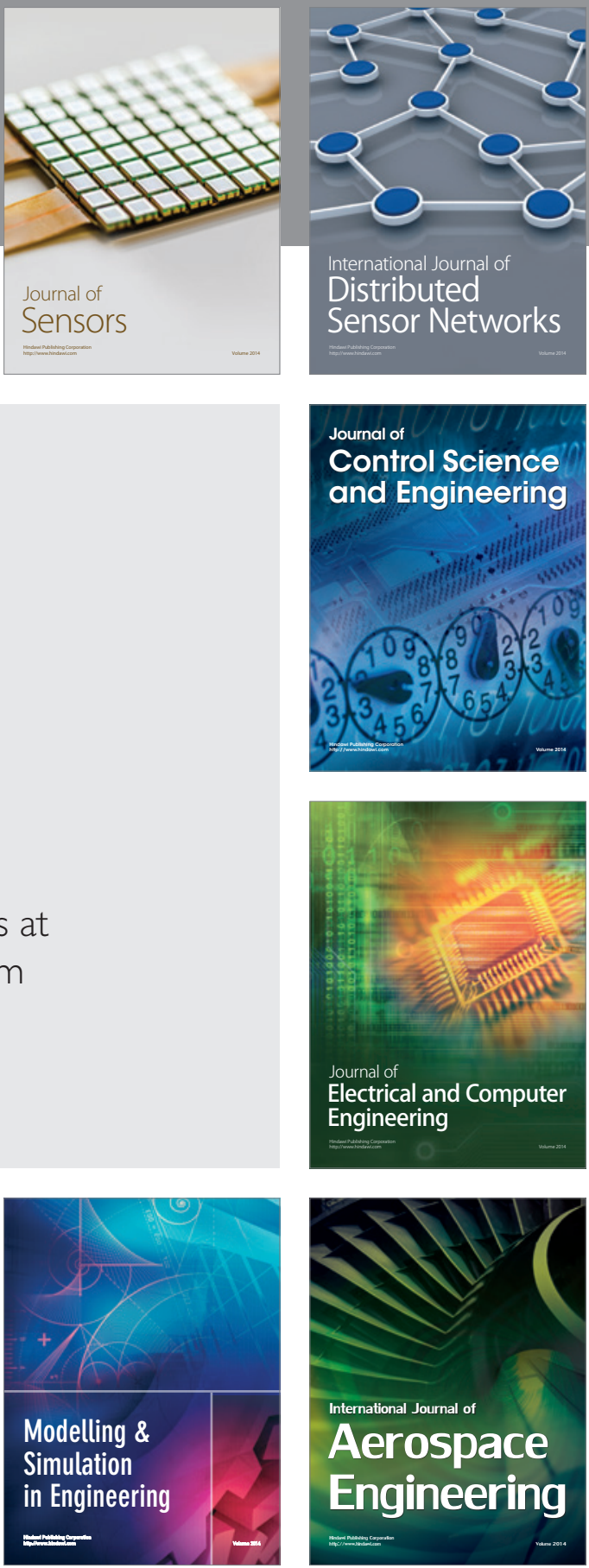

Journal of

Control Science

and Engineering
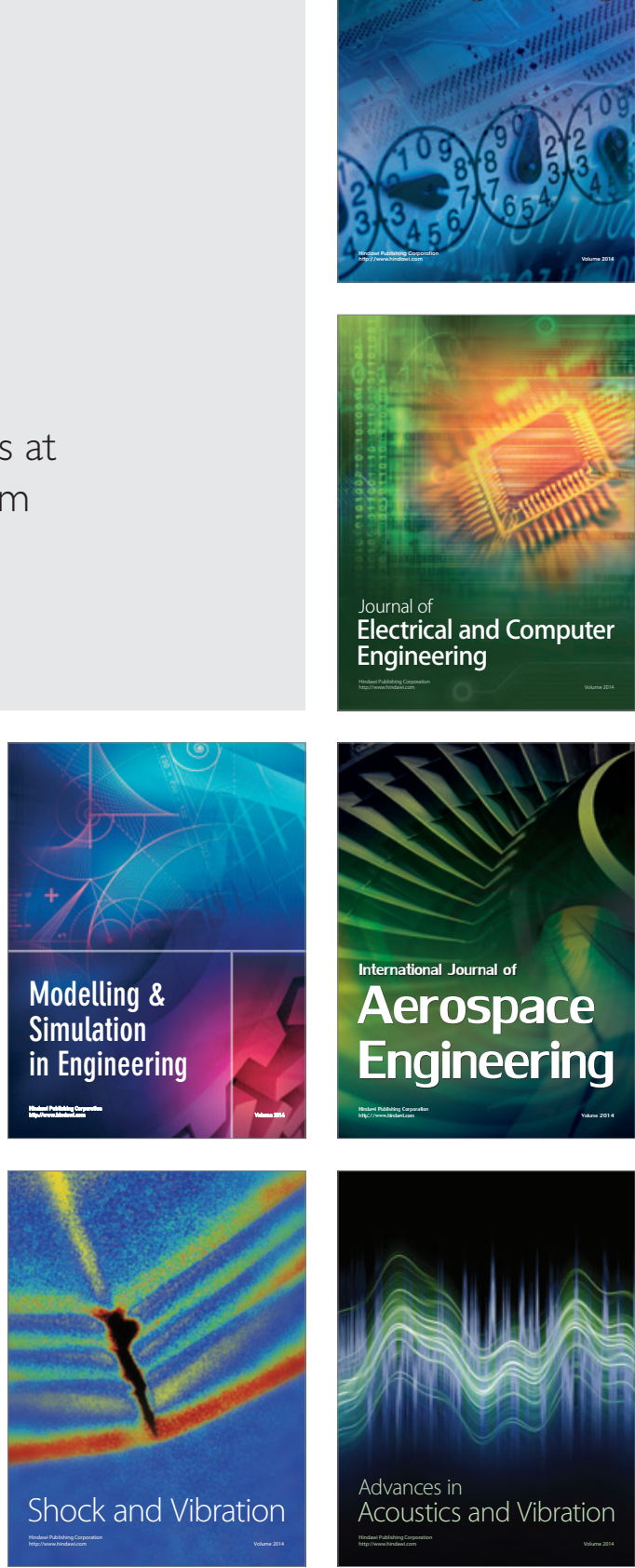